\title{
CHRONIC CONSTIPATION IN CHILDREN. AN UPDATE ON EVALUATION AND MANAGEMENT
}

\author{
Felicia Galos ${ }^{1,2}$, Mihai Stoicescu ${ }^{1,2}$, Mihai Munteanu², Gabriela Nastase ${ }^{2}$, \\ Malina Anghel', Mihaela Balgradean ${ }^{1,2}$ \\ 1 "Carol Davila" University of Medicine and Pharmacy, Bucharest \\ 2 "Marie Curie" Children's Emergency Hospital, Bucharest
}

\begin{abstract}
Constipation is a common problem in childhood, with a children prevalence varying between $0.7 \%$ and $29.6 \%$. Constipation is encountered in all pediatric age groups, with variably severity and duration, from mild and short to severe and chronic forms, with faecal impact and encopresis. This article review the epidemiological, clinical and therapeutically aspects of children constipation, functional in the majority of cases.
\end{abstract}

Keywords: constipation, child

\section{INTRODUCTION}

Constipation is a common phenomenon in pediatric practice. It represents the cause of around $3-5 \%$ of visits to the family physician and approximately $25-30 \%$ of the examinations of the pediatric gastroenterologist. $(1,2)$ Its prevalence is increasing, and it is present, according to various statistics, within $0.7 \%-29.6 \%$ of children (median $8.9 \%$ ). (3) Constipation is found within all age groups, with variable duration and severity, from milder and short-lived cases, to severe and chronic cases with faecal impaction and faecal incontinence (encopresis, soiling). Faecal incontinence is the result of faecal impaction, when liquid stool passes around the hard mass of stool to collect in the rectum and colon (fecaloma). The onset of constipation takes place in the first year of life for $17 \%-40 \%$ of the cases. (4) Except for the neonatal period, the most common form of constipation is functional. Only in $5-10 \%$ of the cases we are dealing with organic constipation, due to malformations of the anus, anomalies of the intestinal neuromuscular structures, endocrine or metabolic diseases, neurologic and neuromuscular diseases, side-reactions to medications, etc.
The pathophysiology of functional constipation is multifactorial. Genetic predisposition, low social status, insufficient intake of fibres, reduced physical activity were all considered as factors that lead to constipation. (5) The most frequent cause of constipation is the decision taken by the child to no longer defecate, after a painful defecation that scared him. (3)

\section{DEFINITION}

The criteria for defining functional constipation are included in the so-called Roma III criteria for gastrointestinal functional disturbances in children and infants, formulated by experts in the field of pediatric gastroenterology and published in 2006 (Table 1). (6,7) Functional constipation in children is defined by the presence of at least two of the following conditions for at least a month in the case of children younger than 4 years (or with a physical development inferior to a normal 4-year-old child), or at least once a week for 2 months in the case of children older than 4 years: reduced evacuative frequency (inferior or equal to two weekly evacuations), at least one weekly faecal incontinence epi- 
sode after sphincter control acquisition, a history of excessive retention of faecal matter or voluntary opposition to defecation, a history of painful defecations or solid faecal matter, the presence of fecaloma or a history of eliminating an increased stool that may clog the toilet bowl. To this, there can be added irritability, loss of appetite and early satiety, associated symptomatology that improves after stool elimination. Abdominal pain is frequent within children with chronic constipation, but it is not a criterion for defining it. (8)

TABLE 1. Roma III diagnostic criteria for functional constipation

In the absence of organic pathology, $\geq 2$ of the following
must occur
For a child with developmental age $<4$
1. $\leq 2$ defecations per week
2. At last 1 episode of incontinence per week after the
acquisition of toilet skills
3. History of excessive stool retention
4. History of painful or hard bowel movements
5. Presence of large fecal mass in the rectum
6. History of large diameter stools that may obstruct the toilet
Accompanying symptoms may include irritability, decreased
appetite and early satiety, which may disappear immediately
following passage of stool.
Criteria fulfilled for at least 1 month before diagnosis
For a child with a developmental age $\geq 4$ years
(with insufficient criteria for irritable bowel syndrome)
1. $\leq 2$ defecations per week
2. At last 1 episode of incontinence per week
3. History of retentive posturing or excessive volitional stool
retention
4. History of painful or hard bowel movements
5. Presence of large fecal mass in the rectum
6. History of large diameter stools that may obstruct the toilet
Criteria fulfilled at least once per week for at least 2 months
before diagnosis

For uniformity in communication intractable constipation and faecal impaction are defined. Therefore, intractable constipation is defined as constipation that does not respond to treatment after 3 months of correctly conducted and observed therapy. Faecal impaction is defined according to the method through which it was recorded: as a palpable mass in the inferior abdominal cavity, identified through physical exam, dilated rectum, full of faecal matter, through rectal examination, namely excessive faecal matter in the distal colon, if the evaluation is made with abdominal x-ray on an empty stomach. $(8,9)$

Recently, guides have been compiled regarding the evaluation and treatment of children with functional constipation, according to their ages, that do not replace clinical judgment, which is fundamental to the diagnosis of functional chronic constipa- tion. (8) These guides' recommendations are valid in the case of children with functional constipation, not in the case of those with constipation determined by medical or surgical causes.

\section{APPROACHING CHILDREN WITH CONSTIPATION}

The majority of children with constipation require a simple diagnostic approach that is based on a correct anamnesis and a thorough objective examination.

The major role of anamnesis and clinical exam is to exclude other pathological conditions in which we encounter defecation difficulties. (8) (Table 2)

TABLE 2. Differential diagnosis of constipation in children

Celiac disease
Hypothyroidism
Hypercalcemia
Hypokalemia
Drugetes mellintus
chemotherapy, heavy metal ingestin)
Vitamin D intoxication
Botulism
Cystic fibrosis
Hirschsprung disease
Anal achalasia
Colonic inertia
Malformations (imperforate anus, anal stenosis)
Pelvic mass (sacral teratoma)
Spinal cord anomalies, trauma
Abnormal abdominal musculature
Pseudoobstruction (neuropathies, myopathies,
mesenchymopaties)
Multiple endocrine

Anamnesis includes meconium emission evaluation, the initial period of constipation and its severity (evacuative frequency, the size and consistency of the stool, the presence of faecal incontinence, pain when defecating, the presence of blood in the stool or on the toilet paper, assuming antalgic postures), the child's growth, the presence of vomit, abdominal pain, abdominal distension, a history of atopic dermatitis. Many times soiled underwear is wrongly identified by parents as diarrhea and not as an involuntary loss of faecal matter, like we encounter in long-term functional constipation, with massive fecaloma.

The age when constipation begins and the moment of meconium emission are among the most important data obtained through anamnesis. The onset of constipation before the child is one-monthold and meconium emission after more than 48 hours from birth raise suspicion of organic consti- 
pation, especially Hirschsprung disease. 99\% of newborns delivered on time eliminate meconium in the first 48 hours of life and $50 \%$ of children with Hirschsprung disease had a delay in eliminating meconium. (10)

Information regarding eating and playtime schedule, playtime duration, school timetable are important, knowing that many times, children caught in a game or an activity will delay the moment of defecation, which determines a retention of faecal matter, conscious at the beginning, which becomes automatic and reflexive with the passing of time. (11)

Special attention will be given to the family anamnesis, the parents' relationship, the relationship between child and parent, the child's temper. A possible abuse of the child, even a sexual one, will be taken into consideration.

An identification of gastrointestinal diseases (Hirschsprung disease, food allergies, intestinal inflammatory diseases, celiac disease), thyroid diseases, parathyroid diseases or cystic fibrosis in the family will be attempted.

Ongoing treatments for other disorders, but also "natura" treatments followed by the child will be analysed for any possible interference with constipation.

The objective examination must be complete, examining systems and apparatuses, a special attention being given to the appraisal of the nutrition status, to the inspection of the abdomen, perineum and the sacral region, to feeling the abdomen and the thyroid gland. Examining the reflexes, the muscle tonus and muscular strength, especially in the lower extremity of the body are included in the clinical evaluation of a child with chronic constipation.

Careful examination of the perineal region provides the recording of anorectal malformations (anteriorized anus, the presence of anal membranes), perianal erythema, anal fissures and, not lastly, signs of spina bifida, such as hyperpigmentation and localized hypertrichosis. Stimulating the external anal sphincter's reflex contraction provides the evaluation of the integrity of the sensorimotor neural pathways of the pelvic floor. Palpating the abdomen may highlight abdominal masses at descendent colon level (fecaloma).

Rectal exploration is recommended for any child with chronic constipation, especially for ones that present alarm signals (Table 3), regardless of age, the exception being cases where there is suspicion of sexual abuse. Digital rectal examination evaluates perianal sensitivity, anal tonus, whether the rectum is dilated or not, the presence of faecal matter in the rectum and its consistency. According to current expert recommendations, digital rectal examination is not mandatory; for the initial functional constipation diagnostic the data obtained through anamnesis and clinical exam being sufficient in the first stage for a diagnostic. (8)

TABLE 3. Alarm signs and symptoms in child with constipation

Constipation starting extremely early in life ( $<1$ month)
Passage of meconium $>48$ hours
Family history for Hirschsprung disease
Ribbon stools
Blood in the stools in the absence of anal fissure
Failure to thrive
Fever
Bilious vomiting
Abnormal thyroid gland
Severe abdominal distension
Perianal fistula
Abnormal position of anus
Absent anal or cremasteric reflex
Decreased lower extremity strength, tone and reflex
Tuft of hair on spine
Sacral dimple
Gluteal cleft deviation
Extreme fear during anal inspection
Anal scars

Normal meconium emission, the late onset of constipation, eliminating increased faecal matter, episodes of faecal incontinence, assuming antalgic postures, the presence of palpable abdominal matter and a rectal ampulla full of faecal matter lead towards the diagnosis of functional constipation.

On the other hand, the early onset of constipation, eliminating the meconium 24-48 hours after birth, an empty rectal ampulla when a rectal examination is performed, frequent obstructive elements, eliminating small "ribbon-shaped" stools or an explosive stool after the rectal examination, are organic constipation characteristics.

Paraclinical investigations are suited to each particular case and are based on the patient's age (younger or not younger than 6 months), on the previous treatments and the patient's response to these treatments, on the facilities of the clinic that the child with constipation goes to. They are not routinely recommended to all children.

Abdominal radiography are useful for recording faecal matter, if the patient is obese, if rectal examination and investigation are refused (especially in cases where there is suspicion of sexual abuse). Instead, it is recommended for children with encopresis that do not present faecal matter in the rectum or the abdomen, in order to evaluate the vertebral column. $(8,12)$ It is not recommended in order to confirm faecal impaction, if the rectal ex- 
amination records a significant accumulation of faecal matter.

Barium enema is recommended in certain specific conditions: in evaluating a patient that is suspected of Hirschsprung disease in order to find the transition area between the aganglionic segment and the normally innervated intestine and the postsurgical evaluation of the rectum of the patient operated for anal atresia or Hirschsprung disease. $(8,12)$ It is rarely recommended in the initial evaluation of chronic constipation patients.

Intestinal transit time that corresponds to eliminating $80 \%$ of the markers administered is generally under 96 hours. If the intestinal transit time is normal, in the presence of encopresis, a psychological evaluation of the child is recommended. $(8,13)$

Studying intestinal transit time is the simplest and most useful method for identifying patients with slow intestinal transit, and is not routinely recommended for defining constipation.

Abdominal ultrasound is not recommended for functional constipation diagnosis. It can evaluate the rectal ampulla diameter, which is increased in the case of children with chronic functional constipation through the accumulation of fecal matter. (8)

Anorectal manometry is recommended when the anamnesis and the physical exam suggest Hirschsprung disease (early onset of constipation, its severity, the absence of encopresis, growth failure, empty rectal ampulla) or when the constipation lingers in spite of correct and rational treatment.

Anorectal manometry evaluates the pressure of the anorectal region and is used to obtain information regarding anorectal functionality. The main indication to perform anorectal manometry is connected to proving the presence of the anal inhibitory reflex that excludes Hirschsprung disease. The anal inhibitory reflex may be absent in the case of children with megarectum. In this case, the differential diagnosis will decided through biopsy, documenting the absence of ganglionary cells, as well as documenting nerve fibre growth for acetylcholinesterase in the case of children with Hirschsprung disease. (14)

The presence of the anal inhibitory reflex excludes Hirschsprung disease, but false positive results can be obtained through the displacement of the sensor, due to the insufflation of the balloon and the recording of the external anal sphincter, and not the interior one.

Various alterations are observed with anorectal manometry on subjects with chronic functional constipation, especially long-term, compared to normal subjects: reduction of the anal inhibitory reflex, increase of the volume that causes the defecation reflex, the lack of relaxation of the external anal sphincter and the pelvic floor during the attempt to defecate (anismus).

In the absence of the anorectal inhibitory reflex a rectal biopsy with histology is necessary.

Rectal biopsy with histological exam is the gold standard when diagnosing Hirschsprung disease. The diagnosis can be accurately determined only if 2-3 biopsies have been made, at $2-3 \mathrm{~cm}$ from the dentate line, preferably obtained through suction, in order to include sufficient mucous. The recommended histological method that either confirms or excludes Hirschprung disease is still controversial. American researchers recommend the use of haematoxylin-eosin coloration due to its availability in all centres, its increased efficiency and its low cost. The presence of one nodule in the submucous excludes Hirschsprung disease. It can be combined with immunohistochemistry that allows demonstrating the growth of nerve fibres for acetylcholine. Other histochemical exams, namely immunohistochemical, in the process of evaluation, are lactate dehydrogenase, namely calretinin. (14)

Colon manometry allows evaluation of the colon mobility in postprandial phase, being useful in diagnosing refractory constipation, but of the functional type, and in diagnosing constipation due to neuromuscular disorders. Colon mobility can be directly studied with the help of colon manometry, or indirectly with the help of segmental transit times. Children with functional constipation, of the "behavioral" retention type have normal colon motility. The only motor alteration is at rectum level which, being dilated for a long time loses elasticity and propulsive contractability. Particularly interesting is the neuronal intestinal dysplasia, which exhibits a similar clinical picture to that of those with Hirschsprung disease. Neuronal intestinal dysplasia can extend to different segments of the colon, on a variable length and it has been found in association with neurofibromatosis and MEN (multiple endocrine neoplay) type IIB syndrome. For the evaluation of these diseases, biopsies at entire colon level are required. (13-15)

Magnetic resonance imaging of the spine is useful for children with non-retention faecal incontinence in recording possible neurological lesions at lumbosacral level.

Colon scintigraphy is recommended in exceptional cases, in evaluating the colon motility of children with constipation and slow intestinal transit. 
Laboratory investigations are indicated in types of constipation that are refractory to treatment and they target the exclusion of allergy, especially that to cow's milk protein, coeliac disease and hypercalcaemia.

Sweat test, to exclude cystic fibrosis, is recommended in cases that are resistant to treatment or with relapses when doses are diminished and for infants with neonatal onset of constipation, after Hirschsprung disease exclusion.

\section{TREATMENT OF CHILDREN WITH CHRONIC FUNCTIONAL CONSTIPATION}

The treatment's goal is to empty the rectal ampulla and to manage the treatment in order for the child to have 1-2 daily painless evacuations. Generally, the children's diet, especially that of those with constipation is weak in fibres, so that increasing the fibre and carbohydrate intake can be sufficient in lighter cases for transit improvement. Fibres act over the intestinal content through mass effect, as well as at small intestine level, provoking the increase of the evacuation speed and frequency. The increase of frequency takes place through two mechanisms: a hydroscopic one (that keeps the water in the intestinal lumen) and another through stimulating bacteria growth.

It is important to note that, regarding children with severe cases of constipation, the supplementary intake of fibres without an emollient treatment of the faecal matter will only aggravate the constipation. Based on experts' opinion, the recommended diet for children with constipation must have a normal content of fibres and liquids. Physical activity, also, must be normal. Regarding educating the child to sit on the potty or the toilet for 5-10 minutes after meals, 2-3 times a day, in a relaxing atmosphere, it must be associated with a dietary and medical treatment, without being aggressively imposed. (7)

The medical treatment (Table 4) includes several medicine groups, of which the most used are faecal matter emollients, such as lactulose and polyethylene glycol (PEG).

Lactulose is effective due to the fact that she is not absorbed in the small intestine and is partially or completely fermented by the colonic saccharolytic bacterial flora, producing fatty acids, hydrogen and carbon dioxide, thus acidifying the faecal $\mathrm{pH}$. In small doses she acts due to the fermentation produced by the bacterial flora, while in bigger doses she acts as osmotic laxatives as well. An unpleasant collateral effect, that may limit its use, is the intestinal meteorism provoked by the produc-
TABLE 4. Laxatives used in chronic constipation treatment in children

\begin{tabular}{|c|c|}
\hline Oral laxatives & Dosages \\
\hline \multicolumn{2}{|l|}{ Osmotic laxatives } \\
\hline Lactulose & $1-2 \mathrm{~g} / \mathrm{kg}$, once or twice/day \\
\hline PEG 3350 & $0.2-0.8 \mathrm{~g} / \mathrm{kg} /$ day (maintenance) \\
\hline PEG 4000 & $1-1.5 \mathrm{~g} / \mathrm{kg} /$ day (fecal disimpaction; $<6$ days) \\
\hline $\begin{array}{l}\text { Magnesium } \\
\text { hydroxide }\end{array}$ & $\begin{array}{l}0.4-1.2 \mathrm{~g} / \text { day }(2-5 \mathrm{y}) ; 1.2-2.4 \mathrm{~g} / \text { day }(6-11 \mathrm{y}) \\
2.4-4.8 \mathrm{~g} / \text { day }(12-18 \mathrm{y})\end{array}$ \\
\hline \multicolumn{2}{|l|}{ Fecal softeners } \\
\hline Mineral oil & $1-3 \mathrm{ml} / \mathrm{kg} /$ day, $\max 90 \mathrm{ml} /$ day $(1-18 \mathrm{y})$ \\
\hline \multicolumn{2}{|l|}{ Stimulant laxatives } \\
\hline Bisacodyl & $5 \mathrm{mg} /$ day $(3-10 \mathrm{y}), 5-10 \mathrm{mg} /$ day (> $10 \mathrm{y})$ \\
\hline Senna & $\begin{array}{l}2.5-5 \mathrm{mg} / \text { day }(2-6 \mathrm{y}), 7.5-10 \mathrm{mg} / \text { day }(6-12 \mathrm{y}), \\
15-20 \mathrm{mg} / \text { day }(>12 \mathrm{y})\end{array}$ \\
\hline Sodiu picosulfate & $\begin{array}{l}2.5-10 \mathrm{mg} / \text { day }(1 \mathrm{mo}-4 \mathrm{y}), 2.5-20 \mathrm{mg} \\
(4-18 \mathrm{y}), \text { once/day }\end{array}$ \\
\hline \multicolumn{2}{|c|}{ Rectal laxatives/enemas } \\
\hline Bisacodyl & $5 \mathrm{mg} /$ day (2-10 y), 5-10 mg (>10 y) \\
\hline Sodium docusate & $60 \mathrm{ml}(<6 \mathrm{y}), 120 \mathrm{ml}(>6 \mathrm{y})$ \\
\hline $\begin{array}{l}\text { Sodium } \\
\text { phosphate }\end{array}$ & $2.5 \mathrm{ml} / \mathrm{kg}, \max 133 \mathrm{ml} /$ dose (1-18 y) \\
\hline $\mathrm{NaCl} 0,9 \%$ & $\begin{array}{l}5 \mathrm{ml} \text { (neonate }<1 \mathrm{~kg}), 10 \mathrm{ml}(>1 \mathrm{~kg}) \\
6 \mathrm{ml} / \mathrm{kg}(>1 \mathrm{y}) \text {, once or twice/day }\end{array}$ \\
\hline Mineral oil & $30-60 \mathrm{ml}(2-11 \mathrm{y}), 60-150 \mathrm{ml}(>11 \mathrm{y})$ \\
\hline
\end{tabular}

PEG = polietilen

tion of gas because of the fermentation. The minimal effective dose may be different for each child.

Recently, to osmotic laxatives there has been added polyethylene glycol (PEG), a polymer of ethylene oxide, soluble, stabile and non-toxic that is not fermented or metabolized by the intestinal flora. PEG acts through the retention of the water with which it is administered, without attracting water from outside the intestine and without an effect on the intestinal motility. It has been demonstrated that PEG is more efficient and better tolerated than lactulose in treating functional constipation and for children under 2 years old. Its long term safety has also been confirmed, a fact that recommends it as the medication of choice for children, if it is available. $(8,16)$

For chronic constipation (with or without encopresis) it is fundamental that a fecaloma softening be performed before initiating the maintenance treatment, with physiological solution or glycerine enemas, or with the help of administering PEG in high doses ( $1.5 \mathrm{~g} / \mathrm{kg} / \mathrm{body})$ for 3-4 days. Not eliminating the fecaloma can determine the paradoxical growth of the encopresis, bloating, abdominal pain, subocclusive phenomena.

In the presence of faecal matter accumulation, with normal celiac and hypothyroidism tests, and an unsatisfying response to therapy or with relapses when decreasing doses, a trial that consists in a diet 
without cow's milk proteins (CMP) can be taken into consideration. Recently, an allergy to cow's milk proteins has been identified as a cause of constipation within children. The mechanisms through which allergic reactions may determine intestinal motility alterations are diverse, among which there is constipation. (18-20)

In exceptional cases, for certain children and adolescents that do not respond to pharmacologic or behavioral therapy, a surgical treatment is recommended (anorectal myotomy, appendectomy with ACE "antegrade continence enema", subtotal colectomy) or alternative treatments, such as biofeedback, electric stimulation or botulinum toxin shots. $(21,22)$

It is known that the presence of constipation may cause psychosocial difficulties for children and adolescents (interrupted relationships and family stress) that last for its entire duration. These children are usually silent, introverts, and they have a tendency to deny their symptomatology.

A good management of constipation may enhance the quality of life of these patients and may

\section{REFERENCES}

1. Mugie S.M., Benninga M.A., Di Lorenzo C. Epidemiology of constipation in children and adults: a systematic review. Best Pract Res Clin Gastroenterol 2011, 25 (1):3-18

2. Benninga M.A. Voskuijl W.P., Taminiau, J.M. Childhood constipation: is there new light in the tunnel? J Pediatr Gastroenterol Nutr, 2004; 39:448-464

3. van den Berg M.M., Benninga M.A., Di Lorenzo C. Epidemiology of children constipation: a systematic review. Am J Gastroenterol 2006. 101(10):2401-2409

4. Mugie S.M., Di Lorenzo C., Benninga M.A. Constipation in childhood. Nat Rev Gastroenterol Hepatol 2011, 8(9);502-511

5. Olaru C., Diaconescu S., Lupu V.V., Gimiga N., Paduraru G., Ignat A., Ciubotariu G., Drug V., Burlea M. Predisposing factors for chronic constipation in children -2 year clinical study. Romanian Journal of Pediatrics, 2014; 63(4):412-415

6. Hyman P., Milla P., Benninga M.A., et al. Childhood functional gastrointestinal disorders: neonate/toddler. Gastroenterology, 2006; 130:1519-1526

7. Rasquin A., Di Lorenzo C., Forbes D., et al. Childhood functional gastrointestinal disorders: child/adolescent. Gastroenterology, 2006; 130:1527-1537

8. Tabbers M.M., Di Lorenzo C., Berger M.Y., et al. Evaluation and treatment of functional constipation in infants and children: Evidencebased recommendations from ESGHAN and NASPGHAN. J Pediatr Gastroenterol Nutr 2014; 58:258-274

9. Olaru C., Diaconescu S., Lupu V.V., Gimiga G., Paduraru G., Ignat A., Olaru R., Drug V., Burlea M. Multidisciplinary approach to chronic constipation associated with fecal incontinence in children. Romanian Journal of Pediatrics 2015; 64(1):14-17

10. Jung P.M. Hirschsprung's disease: one surgeon's experience in one institution. J Pediatr Surg 1995; 30:646-51

11. Milla P., Cucchiara S., Di Lorenzo C., et al. Motility Disorders in childhood: Working Group Report of the First World Congress of Pediatric Gastroenterology, Hepatology and Nutrition. J Pediatr Gastroenetrol Nutr, 2002; 35:s187-s195

12. Reuchlin-Vroklage L., Bierma-Zeistra S.M.A., Benninga M.A. et al. Diagnostic value of abdominal radiography in constipated children: a systematic review. Arch Pediatr Adolesc Med 2005; 159:671-8 prevent associated pathologies, such as recurrent abdominal pain and recurring urinary tract infections.

The demonstrated negative prognostic factors are two: the very young age of symptomatology onset and the presence of encopresis. 1/3 of adolescents with constipation will suffer from constipation at adult age. $(3,23,24)$

\section{SUMMARY}

Constipation is a very common problem in childhood and occurs worldwide. The high prevalence of this condition justifies the investment of resources and educational campaigns directed to its prevention and optimal treatment. It has great psychological impact on children and their families.

This frustrating phenomenon deserves much more attention that it receives to date, although great progress has been made in the past few years in our understanding the physiological and emotional factors associated with childhood constipation.

13. El-Shay M. Chronic idiopathic slow transit constipation pathophysiology and management. Colorect Dis, 2003; 5:288-296

14. Schäppi M.G., Staiano A., Milla P.J., et al. A practical guide for the diagnostic of primary enteric nervous system disorders. J Pediatr Gastroenterol Nutr 2013; 57:677-686

15. Giorgio V., Borrelli O., Smith V.V. et al. High-resolution colonic manometry accurately predicts colonic neuromuscular pathological phenotype in pediatric slow transit constipation. Neurogastroenterol Motil 2013; 25:70-79

16. Candy D., Belsy J. Macrogol (polyetilen glycol) laxatives in children with functional constipation and faecal impaction: a systematic review. Arch Dis Child 2009; 94:156-60

17. Nurko S., Youssef N.N., Sabri M. et al. PEG3350 in treatment of childhood constipation: a multicenter, double-blinded, placebocontrolled trial. J Pediatr 2008; 153:254-61

18. Borrelli O., Barbara G., Di Nardo G. et al. Neuroimmune interaction and anorectal motility in children with food allergy-related chronic constipation. Am J Gastroenterol 2009; 104:454-63

19. Iacono G., Carroccio A., Cavataio F.A. et al. Chronic constipation as a symptom of cow milk allergy. J Pediatr 1995; 126:34-9

20. Irastorza I., Ibanez B., Delgado-Sanzonetti L. et al. Cow's-milk-free diet as a therapeutic option in childhood chronic constipation. J Pediatr Gastroenterol Nutr 2010; 51:171-6

21. Mugie S.M., Machado R.S., Mousa H.M., et al. Ten-year experience using antegrade enemas in children. J Pediatr 2012; 161:700-4

22. Clarke M.C., Chase J.W., Gibb S. et al. Decreased colonic transit time after transcutaneous interferential electrical stimulation in children with slow transit constipation. J Pediatr Surg 2009; 44:408-12

23. Youssef N.N., Langseder A.L., Verga B.J. et al. Chronic childhood constipation is associated with impaired quality of life: a casecontrolled study. J Pediatr Gastroenetrol Nutr, 2005; 41:56-60

24. van Ginkel R., Reitsma J.B., Buller H.A. et al. Chilhood constipation: longitudinal follow-up beyond puberty. Gastroenterology, 2003; 125:357-63 